\title{
Prevalence of Malnutrition and Sarcopenia among Elderly with Low- Energy Femur Fracture. A 6 Months Prospective Analysis of 132 Cases
}

\author{
Nauro Hudson Monteiro1, Diana Borges Dock-Nascimento² and José Eduardo de Aguilar-Nascimento*1 \\ ${ }^{1}$ Health of Sciences Postgraduate Course, University Center of Varzea Grande, Brazil \\ ${ }^{2}$ Health of Sciences Postgraduate Course and Nutrition School of Federal University of Mato Grosso, Brazil
}

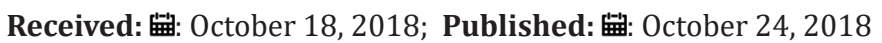

*Corresponding author: José Eduardo de Aguilar-Nascimento, Brazil

\begin{abstract}
Objective: The aim of this study was to assess the prevalence of malnutrition and sarcopenia in elderly patients with low-energy femur fractures. Method: A cross-sectional observational study performed in two public hospitals in Cuiaba-Brazil. Physical examination data were collected from all patients within the first 24 hours after admission. The collection period lasted from May to October 2017. All cases were assessed by SARC-F questionnaire and the nutritional mini-evaluation (NMA). We performed a descriptive and epidemiological analysis for the prevalence of malnutrition and sarcopenia among the patients.
\end{abstract}

Results: One hundred thirty two patients were analyzed, 58.3\% males with mean age of 73.6 (ranging from 60-97) years old. Only 19.7\% (26) of the patients were considered eutrophic and by the SARC-F questionnaire, 64.4\% (85) of the studied population was considered as sarcopenics.

Conclusion: The prevalence of malnutrition and sarcopenia is high in elderly patients with a low-energy femur fracture.

\section{Introduction}

A few studies have reported the correlation between sarcopenia nutritional status with proximal femoral fractures. Ho et al. [1] studied 239 elderly patients with hip fracture and reported that the prevalence of sarcopenia was high and above $70 \%$. Data in this context, such as a profile study of nutritional status and sarcopenia in elderly patients with proximal femoral fracture may improve the information and bring the problem to a better clinical and scientific discussion. It could also raise public awareness about the problem of sarcopenia among the elderly, and to be the basis for the implementation of public health protocols and actions aimed at reducing the prevalence of sarcopenia and, consequently, risk of proximal femur fracture. Thus, the aim of this study is to investigate the prevalence of malnutrition sarcopenia in elderly patients with proximal femur fractures due to low energy accidents.

\section{Material and Methods}

This was a prospective cross-sectional observational clinical study, done in two public hospitals, the St. Benedict Municipal Hospital and Municipal Hospital for Emergencies of Cuiaba, Brazil. Data were collected on the physical examination of the patient, in the first 24 hours of their hospitalization, between May and October of the year 2017. All the research was developed after the approval of the Research Ethics Committee of the Julio Muller Hospital (CEP / HUJM) with the number CAAE: 55047916.3.0000.5541. All patients over 60 years old, with a proximal femoral fracture after a fall were included. An informed consent term was obtained. We exclude patients if they have:

a) Lack of adequate motor coordination to perform the dynamometer test.

b) A previous amputation of a lower limb to perform the anthropometric measurement of calf circumference.

c) Fractures secondary to tumor processes or from high energy trauma.

The nutritional status of the elderly was evaluated by the Mini Nutrition Assessment Reduced Version (MAN) [2] and sarcopenia was assessed by the SARC-F questionnaire and calf circumpherence. SARC-F questionnaire consists of five questions asked directly to the interviewee about their condition before the occurrence of the fracture. The five questions were:

a) Muscular strength 
b) Independence to wander

c) Ability to get up from a chair

d) Ability to climb stairs and finally

e) Their history of falls [3]

The SARC-F questionnaire determines scores of 0 to 2 points for each answer, totaling a maximum of 10 points. Patients with a score equal to or greater than 4 were considered as sarcopenic. The hand grip strength (dynamometry) was performed and the cutoff points were as recommended by the EWGSOP [4] (abnormal: less than 30 $\mathrm{kg}$ for men and less than $20 \mathrm{~kg}$ for women). Calf circumference was measured bilaterally at a point $10 \mathrm{~cm}$ below the anterior tuberosity of the tibia. Cutoff for sarcopenia was less than $33 \mathrm{~cm}$ for women and less than $34 \mathrm{~cm}$ for men [5].

\section{Results}

A hundred fifty-seven patients with proximal femoral fractures were eligible. Twenty-five patients were excluded because due to the exclusion criteria. Thus, 132 patients, 77 males and 55 females, with a mean age of 73 years old, ranging from 60 to 97 years old were studied.

\section{SARC-F}

Only 39 patients $(29.5 \%)$ had a score below 4 (reference value for normality). That is to say that approximately $70 \%$ of the population studied was sarcopenic.

\section{Mini Nutrition Assessment (MAN)}

Twenty-six (19.7\%) patients were considered eutrophic (score equal to or greater than 12 points). Forty-seven cases (35.6\%) were considered as risk of malnutrition and 59 cases $(44.7 \%)$ were considered malnourished [6-7].

\section{Hand Grip Strength}

Abnormal measurements were seen in 62 (80.5\%) of men and in $47(85.5 \%)$ of women. For males, the mean (SD) handgrip strength on both the right side $(22.03 \pm 10.80 \mathrm{~kg})$ and the left side $(21.15 \pm 10.62 \mathrm{~kg})$ were lower than the cutoff point. Likewise, females showed lower means below normality for both the right side $(12.81 \pm 5.85 \mathrm{~kg})$ and left side $(12.30 \pm 6.04 \mathrm{~kg})$ and so, within the diagnostic threshold of sarcopenia.

\section{Calf Circumference}

Abnormal measurements were seen in 61 men and in 50 women. These data show that $79.2 \%$ of men and $90.9 \%$ of women were sarcopenic by this parameter.

\section{Discussion}

The overall results observed in the present study show that elderly patients with proximal femoral fractures are potentially malnourished and have a high incidence of sarcopenia. We found evidence of this sarcopenia in both the SARC- F questionnaire and the handgrip strength. These data are highly suggestive of the fact that malnourished and sarcopenia elderly patients are at great risk of being affected by falls and therefore present with bone fractures such as the proximal femur. In the medical literature, proximal femoral fracture has been markedly associated with osteoporosis $[6,7]$ but most of the studies did not focused on sarcopenia. Our data show that care should be given in the same way to the nutritional status of the patient and their degree of sarcopenia.

\section{Conclusion}

The observed results allow us to conclude that the prevalence of malnutrition and sarcopenia is high among elderly people with proximal femoral fractures due to low energy. These two factors should be focused in further studies to improve the knowledge of predictors factors for proximal femur fractures in elderly.

\section{References}

1. Ho AW, Lee MM, Chan EW, Ng HM, Lee CW, et al. (2016) Prevalence of pre sarcopenia and sarcopenia in Hong Kong Chinese geriatric patients with hip fracture and its correlation with different factors. Hong Kong Med J 22(1): 23-29.

2. Hudgens J, Langkamp Henken B (2004) The Mini Nutritional Assessment as an assessment tool in elders in long term care. Nutr Clin Pract 19(5): 463-470.

3. Malmstrom TK, Miller DK, Simonsick EM, Ferrucci L, Morley JE (2016) SARC-F: a symptom score to predict persons with sarcopenia at risk for poor functional outcomes. J Cachexia Sarcopenia Muscle 7(1): 28-36.

4. Cruz Jentoft AJ, Baeyens JP, Bauer JM, Boirie Y, Cederholm T, et al. (2010) European Working Group on Sarcopenia in Older People. Sarcopenia: European consensus on definition and diagnosis: Report of the European Working Group on Sarcopenia in Older People. Age ageing 39(4): 412423.

5. Rolland Y, Lauwers Cances V, Cournot M, Nourhashémi F, Reynish W, Rivière D, et al. (2003) Sarcopenia, calf circumference and physical function of elderly women: a cross sectional study. J Am Geriatr Soc 51(8): 1120-1124.

6. Aldieri A, Terzini M, Osella G, Priola AM, Angeli A, et al. (2018) Osteoporotic hip fracture prediction: is T score based criterion enough? A Hip Structural Analysis based model. J Biomech Eng. Jun 25.

7. Dragomir Daescu D, Rossman TL, Rezaei A, Carlson KD, Kallmes DF, et al. (2018) Factors associated with proximal femur fracture determined in a large cadaveric cohort. Bone 116: 196-202. 


\section{ISSN: 2574-1241}

DOI: 10.26717/BJSTR.2018.10.001935

José Eduardo de Aguilar-N. Biomed J Sci \& Tech Res

(c) (i) This work is licensed under Creative

Submission Link: https://biomedres.us/submit-manuscript.php

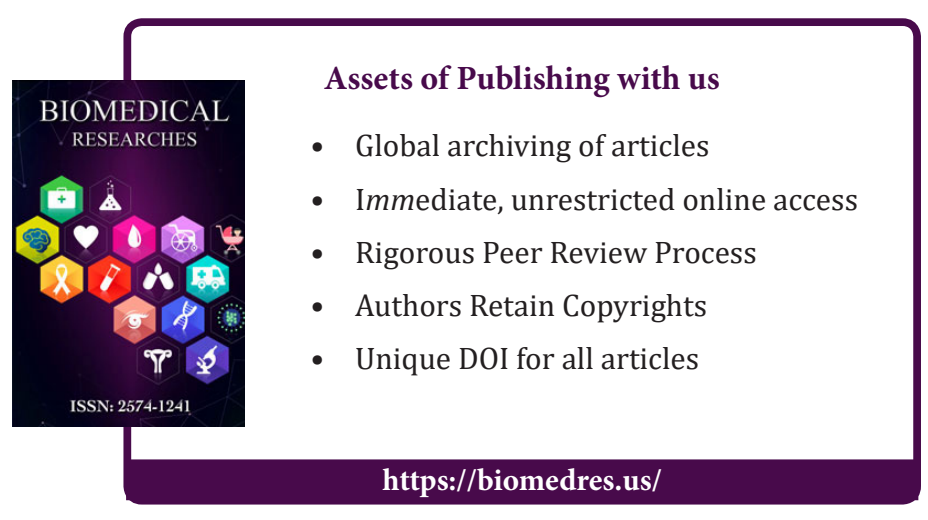

